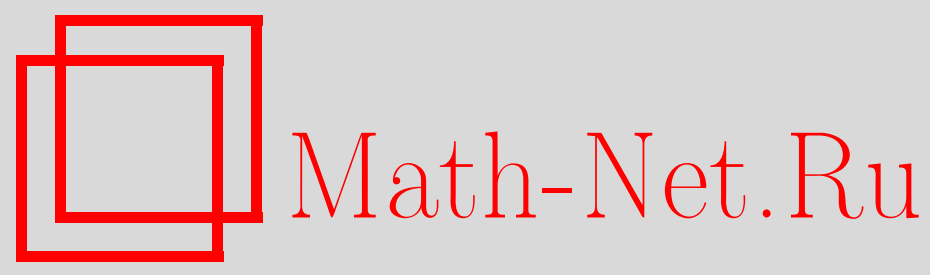

М. И. Граев, Относительно замкнутые операторы, связанные с парой грассманианов, Матем. заметки, 2002, том 71, выпуск 1, 61-74

DOI: https://doi.org/10.4213/mzm328

Использование Общероссийского математического портала Math-Net.Ru подразумевает, что вы прочитали и согласны с пользовательским соглашением http://www . mathnet.ru/rus/agreement

Параметры загрузки:

IP: 34.227 .88 .159

26 апреля 2023 г., $15: 20: 43$

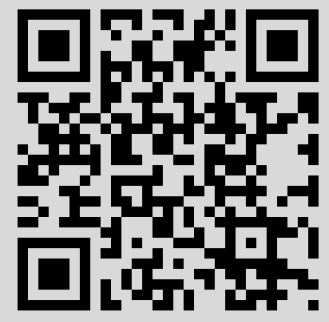




\title{
ОТНОСИТЕЛЬНО ЗАМКНУТЫЕ ОПЕРАТОРЫ, СВЯЗАННЫЕ С ПАРОЙ ГРАССМАНИАНОВ
}

\author{
М.И. Граев
}

Понятие относительо замкнутых операторов естественно возникает в задачах интегральной геометрии при построении локальных формул обращения. В статье построены семейства относительно замкнутых операторов для интегральных преобразований, связанных с парами вещественных грассманианов. Как следствие получено описание всех локальных формул обращения для этих преобразований.

Библиограффия: 7 названий.

Введение. Изложим, не приводя точных формулировок, определение относительной замкнутости в интегральной геометрии.

Пусть $X, Y$ - многообразия, между которьми задано отношение инцидентности $A \subset$ $X \times Y ; X^{y} \subset X$ - подмногообразие точек $x \in X$, инцидентных одной точке $y \in Y$; аналогично определяются подмногообразия $Y^{x}$ в $Y$. Предполагается, что отношение инцидентности удовлетворяет набору естественных требований (см. [1]), а на подмногообразиях $X^{y}$ заданы меры. Обозначим через $\mathscr{J} f(y)$ интеграл функции $f$ на $X$ по подмногообразию $X^{y}$. Таким образом, отображение $\mathscr{J}$ преобразует функции на $X$ в функции на $Y$. (На самом деле, вместо функций на $X$ и $Y$ часто рассматриваются сечения подходящих расслоений над $X$ и $Y$.)

Пусть $\kappa$ - линейный дифференциальньй оператор, переводящий функции на $Y$ во внешние формы степени $p<\operatorname{dim} Y^{x}$ на $Y$. Скажем, что $\kappa$ относительно замкнут, если

$$
\left.d \kappa(\mathscr{J} f)\right|_{Y^{x}}=0
$$

для любой функции $f$ на $X$ и любой точки $x \in X$ (см. примеры в [1], [2]).

Если $\kappa$ относительно замкнут, то для любой точки $x \in X$ и любого цикла $\gamma^{x} \subset Y^{x}$ размерности $p$ имеем

$$
\int_{\gamma^{x}} \kappa(\mathscr{J} f)=c\left(\gamma^{x}\right)(L f)(x),
$$

где постоянная $c\left(\gamma^{x}\right)$ зависит только от класса гомологий $\gamma^{x}$, а $L$ - линейньй дифференщиальный оператор, зависящий только от $\kappa$; например, $L$ - оператор умножения на

Работа выполнена при поддержке Российского фонда фундаментальных исследований, грант № 98-01-00798. 
функцию от $x$. Оператор $\kappa$ назьвается тривиальным, если правая часть (1) тождественно равна 0. Таким образом, для каждого нетривиального относительно замкнутого оператора $\kappa$ равенство (1) восстанавливает $L f$ по образу $\mathscr{J} f$ функции $f$.

Эта статья посвящена описанию относительно замкнутых операторов $\kappa$ для пары $X=G_{l, n}, Y=G_{k, n}$, где $G_{l, n}, G_{k, n}-$ многообразия (грассманианы) соответственно $l$-мерных и $k$-мерных ориентированных подпространств в $\mathbb{R}^{n}, l<k<n-l$, и $A \subset X \times Y$-многообразие флагов. Явно построены все, с точностью до тривиальных, относительно замкнутые операторы $\kappa$, для которых $L$ является умножением на функцию. Пространство этих операторов наделено естественной структурой $G L(n)$-модуля, и как $G L(n)$-модуль оно изоморфно пространству $S^{k} \bigwedge^{l} \mathbb{C}^{n}$, где $S^{k}$ и $\bigwedge^{l}$ - соответственно симметрическая и внешняя тензорные степени. Случай $l=1$ был ранее исследован в [3].

Одновременно построен класс операторов $\kappa$, удовлетворяющих условию относительной замкнутости более общего вида

$$
\left.d \kappa(\mathscr{J} f)\right|_{G_{k, n}^{h}}=0 \quad \text { для любого } h \in G_{r, n},
$$

где $l \leqslant r<k, G_{k, n}^{h} \subset G_{k, n}$ - подмногообразие $k$-подпространств, содержащих $h$. Этим операторам отвечают формулы обращения, восстанавливающие по функции $\mathscr{J} f$ интегралы исходной функции $f$ по подмногообразиям $l$-мерных подпространств, принадлежащих произвольному фиксированному $r$-мерному подпространству (см. [4], [5]). Подробному исследованию таких операторов и связанных с ними формул обращения будет посвящена другая статья.

1. Интегральные преобразования, связанные с парами грассманианов. Изложим определение из [6] и основные свойства интегрального преобразования $\mathscr{J}_{k l}$, связанного с многообразиями (грассманианами) $G_{l, n}$ и $G_{k, n} l$-мерных и $k$-мерных ориентированных подпространств пространства $\mathbb{R}^{n}$, где $l<k$.

Пусть $\mathscr{E}_{k, n}, k<n,-$ многообразие вешественньх невырожденных $(k \times n)$-матриц. На $\mathscr{E}_{k, n}$ транзитивно действует групш $G L(k) \times G L(n)$ согласно формуле $x \in \mathscr{E}_{k, n} \mapsto g_{1}^{-1} x g_{2}$ для каждой пары $\left(g_{1}, g_{2}\right) \in G L(k) \times G L(n)$. Многообразие $\mathscr{E}_{k, n}$ является главным расслоенным пространством группы $G L_{+}(k)$ над грассманианом $G_{k, n}:$ проекция $\mathscr{E}_{k, n} \rightarrow$ $G_{k, n}=G L_{+}(k) \backslash \mathscr{E}_{k, n}$ относит каждой матрице $x \in \mathscr{E}_{k, n}$ ориентированное подпространство, натянутое на ее векторы-строки. Очевидно, отображение $\mathscr{E}_{k, n} \rightarrow G_{k, n}$ перестановочно с действием групшы $G L(n)$ на $\mathscr{E}_{k, n}$ и $G_{k, n}$.

Обозначим для любой пары натуральных чисел $l, k \leqslant n$ через $H_{l}^{k}$ подпространство $C^{\infty}$-функций на $\mathscr{E}_{l, n}$, удовлетворяющих условию

$$
f(g x)=|\operatorname{det} g|^{-k} f(x) \quad \text { для любого } g \in G L(l) \text {. }
$$

Заметим, что элементы из $H_{l}^{k}$ можно интерпретировать как сечения одномерного расслоения над грассманианом $G_{l, n}$

Свяжем с каждой функцией $f \in H_{l}^{k}$ и каждой точкой $x \in E_{l, n}$ следующую дифференциальную форму степени $l(k-l)$ на многообразии $\mathscr{E}_{l, k}$ :

$$
f(t x) \sigma_{l k}(t)=f(t x) \sigma_{l k}^{1}(t) \wedge \cdots \wedge \sigma_{l k}^{l}(t)
$$


где $\sigma_{l k}^{j}(t)=\sum \operatorname{sign}\left(i_{1}, \ldots, i_{k}\right) t_{1}^{i_{1}} \cdots t_{l}^{i_{l}} d t_{j}^{i_{l+1}} \wedge \cdots \wedge d t_{j}^{i_{k}}$ (сумма по всем перестановкам $\left(i_{1}, \ldots, i_{k}\right)$ индексов $\left.1, \ldots, k\right)$. Эта форма опускается с многообразия $\mathscr{E}_{l, k}$ на грассманиан $G_{l, k}$, а потому ее интеграл по любому сечению расслоения $\mathscr{E}_{l, k} \rightarrow G_{l, k}$ не зависит от выбора этого сечения.

По определению интегральное преобразование, связанное с грассманианами $G_{l, n}$ и $G_{k, n}$, где $l<k \leqslant n-l$, есть оператор $\mathscr{J}_{k l}: H_{l}^{k} \rightarrow H_{k}^{l}$, заданный следующей формулой:

$$
\left(\mathscr{J}_{k l} f\right)(x)=\int_{G_{l, k}} f(t x) \sigma_{l k}(t) .
$$

Оператор $\mathscr{J}_{k l}$ перестановочен с представлениями группы $G L(n)$, естественно действуюшими на пространствах $H_{l}^{k}$ и $H_{k}^{l}$, и можно доказать, что этим свойством он определяется однозначно с точностью до множителя.

В [6] доказано, что функция $\varphi \in H_{k}^{l}$ принадлежит образу оператора $\mathscr{J}_{k l}$ тогда и только тогда, когда она удовлетворяет следующей системе дифференциальных уравнений степени $l+1$ :

$$
\operatorname{det}\left\|\partial_{s_{j}}^{\nu_{i}}\right\|_{i, j=1, \ldots, l+1} \varphi=0 \text { для любых } \nu_{s}=1, \ldots, k, \quad j_{t}=1, \ldots, n,
$$

где $\partial_{j}^{\nu}=\partial / \partial x_{\nu}^{j}, x_{\nu}^{j}, j=1, \ldots, n, \nu=1, \ldots, k,-$ элементы матриц $x \in \mathscr{E}_{k, l}$.

Условиям (1.3) можно придать другую эквивалентную форму, введя следующие операторы на $\mathscr{E}_{k, n}$ :

$$
d_{j}^{i}=\sum_{s=1}^{n} \partial_{s}^{i} d x_{j}^{s}, \quad i, j=1, \ldots, k
$$

Именно,

$$
\sum_{j}\left(d_{j_{1}}^{i_{1}} \wedge \cdots \wedge d_{j_{l+1}}^{i_{l+1}}\right) \varphi=0
$$

где сумма берется по всем перестановкам индексов $j_{1}, \ldots, j_{l+1}$.

В частности,

$$
\left(d_{j}^{i_{1}} \wedge \cdots \wedge d_{j}^{i_{l+1}}\right) \varphi=0 \quad \text { для любых } i_{1}, \ldots, i_{l+1}, j=1, \ldots, k .
$$

При $k+l<n$ и четном $k-l$ в [6] получена формула обращения, восстанавливающая функцию $f \in H_{l}^{k}$ по ее образу $\mathscr{J}_{k l} f$. Приведем ее в удобной для дальнейшего форме. Введем оператор на $H_{k}^{l}$ :

$$
\kappa_{l}=\bigwedge_{j=1}^{k-l} \bigwedge_{i=1}^{l} d_{r+j}^{i}
$$

Отождествим $\mathscr{E}_{k, n}$ с открытьм подмножеством в $\mathscr{E}_{l, n} \times \mathscr{E}_{k-l, n}$, полагая $x=\left(x^{\prime}, x^{\prime \prime}\right)$, где $x^{\prime} \in \mathscr{E}_{l, n}$ и $x^{\prime \prime} \in \mathscr{E}_{k-l, n}$ состоят соответственно из первых $l$ и последних $k-l$ строк матрицы $x \in \mathscr{E}_{k, n}$. Обозначим через $\mathscr{E}_{k, n} x^{\prime}, x^{\prime} \in \mathscr{E}_{l, n}$, подмногообразие матрищ $x=\left(x^{\prime}, x^{\prime \prime}\right) \in \mathscr{E}_{k, n}$ с фиксированным $x^{\prime}$.

Доказано, что для любой функции $\varphi \in H_{k}^{l}$ и любого $x^{\prime} \in \mathscr{E}_{l, n}$ дифференциальная форма $\left.\kappa \varphi\right|_{\mathscr{E} x_{k, n}^{\prime}}$ опускается на грассманиан $G_{k-l, n}$ при естественном отображении $\mathscr{E}_{k, n}^{x^{\prime}} \rightarrow G_{k-l, n}$ 
ТЕорема. Если $\varphi=\mathscr{J}_{k l} f, f \in H_{l}^{k}$, то для любого $x^{\prime} \in \mathscr{E}_{l, n}$ дифференииальная форма $\kappa_{l} \varphi$ замкнута на $\mathscr{E}_{k, n}^{x^{\prime}}$ u

$$
\int_{\gamma} \kappa_{l} \varphi=c(\gamma) f\left(x^{\prime}\right)
$$

для любого иикла $\gamma$ размерности $l(k-l)$ в проекции $\mathscr{E}_{k, n}^{x^{\prime}}$ на $G_{k-l, n}$. Здесь $c(\gamma)$ зависит только от класса гомологий $\gamma$ и $c(\gamma) \not \equiv 0$; например, $c(\gamma) \neq 0$, если $\gamma-$ подмногообразие $(k-l)$-мерных подпространств, принадлежащих фиксированному $k$-мерному подпространству.

2. Условие относительной замкнутости. Пусть $\kappa$ - линейньй дифференциальный оператор из пространства функций на $\mathscr{E}_{k, n}$ в пространство дифференциальных форм произвольной фиксированной степени на грассманиане $G_{k, n}$. Скажем, что $\kappa$ удовлетворяет условию относительной замкнутости типа $r$, где $l \leqslant r<k$ (короче, относительно замкнут типа $r$ ), если для любой функции $\varphi \in H_{k}^{l}$ из образа интегрального преобразования $\mathscr{J}_{k l}$ и любого $h \in G_{r, n}$ дифференциальная форма $\kappa \varphi$ замкнута на подмногообразии $G_{k, n}^{h} \subset G_{k, n} k$-подпространств, содержащих $h$.

Таким образом, с каждым относительно замкнутым оператором $\kappa$ типа $r$ связано пространство замкнутых дифференциальных форм $d \kappa\left(\mathscr{J}_{k l} f\right), f \in H_{l}^{k}$, на $G_{k, n}$, равных нулю на подмногообразиях $G_{k, n}^{h}, h \in G_{r, n}$.

Если $\kappa$ - относительно замкнутый оператор типа $r$ и $\operatorname{deg}(\kappa \varphi)<\operatorname{dim} G_{k, n}^{h}$, то для каждого $h \in G_{r, n}$ линейный функционал на $H_{l}^{k}$

$$
f \mapsto \int_{\gamma^{h}} \varphi\left(\mathscr{J}_{k l} f\right)
$$

где $\gamma^{h}$ - цикл в $G_{k, n}^{h}$ размерности $\operatorname{deg}(\kappa \varphi)$, зависит только от класса гомологий $\gamma^{h}$ и сосредоточен на подмногообразии $l$-подпространств, содержащихся в $h$. В частности, при $r=l$ он сосредоточен в точке $h$. Назовем $\kappa$ тривиальным, если для каждого $\gamma^{h}$ этот линейный функционал равен тождественно нулю.

Здесь для каждого $r$ из интервала $l \leqslant r<k$ будут явно построены семейства относительно замкнутых операторов $\kappa$ типа $r$.

Сначала введем алгебру $A_{k}$ линейных операторов из пространства функций на $\mathscr{E}_{k, n}$ в пространство дифференциальных форм на $\mathscr{E}_{k, n}$ :

$$
A_{k}=\mathscr{D} \otimes \Omega
$$

где $\mathscr{D}$ - алгебра линейных дифференциальных операторов с полиномиальными коэффициентами на $\mathscr{E}_{k, n}$, a $\Omega$ - внешняя алгебра дифференциальных форм на $\mathscr{E}_{k, n}$ с постоянньми коэффициентами. По определению оператор $\kappa=\partial \otimes \omega$, где $\partial \in \mathscr{D}, \omega \in \Omega$, действует на функции $\varphi$ по формуле $\kappa \varphi=(\partial \varphi) \omega$. Степень дифференциальной формы $\kappa \varphi$ назовем рангом оператора $\kappa$.

Алгебра $A_{k}$ снабжена естественной градуировкой:

$$
A_{k}=\bigoplus_{p, q}\left(\mathscr{D}_{p} \times \Omega_{q}\right)
$$


где $\mathscr{D}_{p}$ - пространство однородных линейных дифференциальных операторов степени $p$ на $\mathscr{E}_{k, n}$ с полиномиальньми коэффициентами и $\Omega_{q}-$ пространство дифференциальных форм степени $q$ на $\mathscr{E}_{k, n}$ с постоянными коэффициентами. В пространствах $\mathscr{D}_{p}, \Omega_{q}$ и на всем пространстве $A_{k}$ определены представления группы $G L(k) \times G L(n)$, порожденные действием этой группы на многообразии матриц $\mathscr{E}_{k, n}$. Очевидно, что пространство $A_{k}$ разлагается в прямую сумму инвариантных конечномерных неприводимых подпространств.

Обозначим через $A_{k}^{l}$ подпространство операторов $\kappa \in A_{k}$ таких, что для любой функции $\varphi \in H_{k}^{l}$ дифференциальная форма $\kappa \varphi$ опускается с $\mathscr{E}_{k, n}$ на $G_{k, n}$, а потому может рассматриваться как форма на грассманиане $G_{k, n}$. Обозначим далее через $B_{k}^{l, r} \subset A_{k}^{l}$ подпространство относительно замкнутых операторов типа $r$, где $l \leqslant r<k$. Очевидно, что подпространства $A_{k}^{l}$ и $B_{k}^{l, r}$ инвариантны относительно группы $G L(k) \times G L(n)$ и подобно всему пространству $A_{k}$ они разлагаются в прямую сумму инвариантных конечномерных неприводимых подпространств.

Каждому оператору $\kappa \in B_{k}^{l, r}$ типа $r$ и каждому $h \in G_{r, n}$ соответствует линейный функционал на $H_{l}^{k}$ :

$$
f \mapsto \int_{\gamma^{h}} \kappa\left(\mathscr{J}_{k l} f\right)
$$

где $\gamma^{h}$ - цикл подходящей размерности в подмногообразии $G_{k, n}^{h} \subset G_{k, n} k$-подпространств, содержащих $h$. Он зависит только от класса гомологий $\gamma^{h}$ и сосредоточен на подмногообразии $l$-подпространств, принадлежащих $h$. В частности, при $r=l$ он сосредоточен в точке $l \in \mathscr{E}_{l, n}$. Назовем $\kappa$ тривиальным, если для любого цикла $\gamma$ этот функционал равен тождественно нулю.

3. Операторы $\sigma(a, \alpha ; p, q)$. Этот и следующий пункты посвящены построению операторов $\kappa \in A_{k}^{l}$. Воспользуемся следующими обозначениями (см. [7]). Пусть заданы наборы $f^{i}=\left(f_{1}^{i}, \ldots, f_{k}^{i}\right), i=1, \ldots, k-p$, и $\omega^{i}=\left(\omega_{1}^{i}, \ldots, \omega_{k}^{i}\right), i=k-p+1, \ldots, k$, где $f_{j}^{i}$ - функции, а $\omega_{j}^{i}$ - дифференциальные 1-формы с операторньгми коэффициентами. Положим по определению

$$
\left[f^{1}, \ldots, f^{k-p}, \omega^{k-p+1}, \ldots, \omega^{k}\right]=\sum \operatorname{sign}\left(j_{1}, \ldots, j_{k}\right) f_{j_{1}}^{1} \cdots f_{j_{k-p}}^{k-p} \omega_{j_{k-p+1}}^{k-p+1} \wedge \cdots \wedge \omega_{j_{k}}^{k},
$$

сумма берется по всем перестановкам $\left\{j_{1}, \ldots, j_{k}\right\}$ индексов $1, \ldots, k$. Предполагается, что операторы, входящие в $\omega_{j}^{i}$, не действуют на коэффициенты последующих сомножителей, например

$$
\left(a(x) \frac{\partial}{\partial x_{1}} d x_{1}\right) \wedge\left(b(x) \frac{\partial}{\partial x_{2}} d x_{2}\right)=a(x) b(x) \frac{\partial^{2}}{\partial x_{1} \partial x_{2}} d x_{1} \wedge d x_{2} .
$$

Выражение (3.1) можно записать в виде детерминанта

$$
\left|\begin{array}{cccccc}
f_{1}^{1} & \ldots & f_{1}^{k-p} & \omega_{1}^{k-p+1} & \ldots & \omega_{1}^{k} \\
\vdots & & \vdots & \vdots & & \vdots \\
f_{k}^{1} & \ldots & f_{k}^{k-p} & \omega_{k}^{k-p+1} & \ldots & \omega_{k}^{k}
\end{array}\right|
$$


при раскрытии которого дифференциальные формы перемножаются внешним образом. Из определения следует, что этот детерминант антисимметричен относительно любых двух строк и относительно первых $k-p$ столбцов и симметричен относительно последних $p$ столбцов.

Введем операторы

$$
\begin{aligned}
& {\left[x^{r_{1}}, \ldots, x^{r_{\alpha}}, d x^{i_{1}}, \ldots, d x^{i_{\beta}}, D^{j_{1}}, \ldots, D^{j_{\gamma}}\right]} \\
& \quad=\sum_{s}\left[x^{r_{1}}, \ldots, x^{r_{\alpha}}, d x^{i_{1}}, \ldots, d x^{i_{\beta}}, d x^{s_{1}}, \ldots, d x^{s_{\gamma}}\right] E_{s_{1}}^{j_{1}} \circ \ldots \circ E_{s_{\gamma}}^{j_{\gamma}}
\end{aligned}
$$

где $\alpha+\beta+\gamma=k$. Здесь обозначено

$$
\begin{gathered}
E_{s}^{i}=\sum_{\nu=1}^{k} x_{\nu}^{i} \partial_{s}^{\nu}, \quad \text { где } \partial_{s}^{\nu}=\frac{\partial}{\partial x_{\nu}^{s}}, i, s=1, \ldots, n, \\
D_{j}^{i}=\sum_{s=1}^{n} E_{s}^{i} d x_{j}^{s}=\sum_{s=1}^{n} \sum_{\nu=1}^{k} x_{\nu}^{i} \partial_{s}^{\nu} d x_{j}^{s} .
\end{gathered}
$$

Кружочки вместо знаков умножения означают, что операторы не действуют на коэффициенты последующих операторов, т.е.

$$
E_{s_{1}}^{j_{1}} \circ \cdots \circ E_{s_{\gamma}}^{j_{\gamma}}=\sum_{\nu} x_{\nu_{1}}^{j_{1}} \cdots x_{\nu_{\gamma}}^{j_{\gamma}} \partial_{s_{1}}^{\nu_{1}} \cdots \partial_{s_{\gamma}}^{\nu_{\gamma}}
$$

Рассмотрим семейство операторов, представимых как внешние произведения $l$ операторов вида (3.2). Для их описания используем несколько обозначений. Обозначим через $\mathscr{A}$ множество пар $(a, \alpha)$, где $a-(l \times n)$-матрица с элементами 0 и 1 такая, что $\sum_{j=1}^{n} a_{i j} \leqslant k, i=1, \ldots, l, \alpha=\left(\alpha_{1}, \ldots, \alpha_{n}\right)$ - последовательность целых неотрицательных чисел такая, что $\sum_{j=1}^{n} \alpha_{j}=k l-\sum_{i, j} a_{i j}$.

Свяжем с каждой парой $(a, \alpha) \in \mathscr{A}$ множество $U=U(a, \alpha)$ пар $(l \times n)$-матриц $p=\left\|p_{i j}\right\|$ и $q=\left\|q_{i j}\right\|$ с цельми неотрицательными элементами, удовлетворяющих следуюшим условиям:

$$
\sum_{j=1}^{n}\left(a_{i j}+p_{i j}+q_{i j}\right)=k, \quad i=1, \ldots, l, \quad \sum_{i=1}^{l}\left(p_{i j}+q_{i j}\right)=\alpha_{j}, \quad j=1, \ldots, n .
$$

В частности, если $\alpha_{j}=0$ для какого-либо $j$, то $p_{i j}=q_{i j}=0$ для любого $i=1, \ldots, l$. Отметим, что вместе с каждой парой $(p, q)$ множество $U(a, \alpha)$ содержит также пары $\left(p-e_{i j}, q+e_{i j}\right)$ при $p_{i j}>0$ и пары $\left(p-e_{i j}, q+e_{i j^{\prime}}+e_{i^{\prime} j}-e_{i^{\prime} j^{\prime}}\right)$ при $p_{i j}>0$ и $q_{i^{\prime} j^{\prime}}>0$. Здесь через $e_{i j}$ обозначена $(l \times n)$-матрица с единицей на $(i, j)$-м месте и нулями на остальных местах.

Определим для любых $(a, \alpha) \in \mathscr{A}$ и $(p, q) \in U(a, \alpha)$ оператор $\sigma(a, \alpha ; p, q) \in A_{k}$ формулой

$$
\sigma(a, \alpha ; p, q)=\bigwedge_{i=1}^{l} \sigma_{i}(a, \alpha ; p, q)
$$


где $\sigma_{i}(a, \alpha ; p, q)$ - оператор (3.2), у которого

1) последовательность $r_{1}, \ldots, r_{\alpha}$ совпадает с возрастающей последовательностью индексов $j$, для которых $a_{i j}=1$;

2 ) последовательность $i_{1}, \ldots, i_{\beta}$ содержит каждый индекс $j=1, \ldots, n$ с кратностью $p_{i j}$

3) последовательность $j_{1}, \ldots, j_{\gamma}$ содержит каждый индекс $j=1, \ldots, n$ с кратностью $q_{i j}$.

Предполагается, что дифференциальные операторы $\partial_{\nu}^{s}$, входящие в выражение для $\sigma(a, \alpha ; p, q)$, не действуют на коэффищиенты последующих операторов.

Из определения следует, что $\sigma(a, \alpha ; p, q) \in \mathscr{D}_{s} \otimes \Omega_{t}$, где $s=|q|=\sum_{i, j} q_{i j}, t=\sum_{j=1}^{n} \alpha_{j}$.

Выражение для $\sigma(a, \alpha ; p, q)$ можно представить в другой форме.

ПРЕДЛОЖЕНИЕ 1. Если $\left\{D^{j_{1}}, \ldots, D^{j_{N}}\right\}$ - последовательность всех столбиов $D^{j}$, входящих в выражсение (3.4) для $\sigma(a, \alpha ; p, q)\left(N=|q|=\sum_{i, j} q_{i j}\right)$, то

$$
\sigma(a, \alpha ; p, q)=\sum_{s} E_{s_{1}}^{j_{1}} \circ \cdots \circ E_{s_{N}}^{j_{N}} \widetilde{\sigma}(a, \alpha ; p, q)
$$

где $\widetilde{\sigma}(a, \alpha ; p, q)$ получается из $\sigma(a, \alpha ; p, q)$ заменой каждого столбца $D^{j_{\nu}}$ на столбeu, $d x^{s_{\nu}}$.

4. Построение операторов $\kappa \in A_{k}^{l}$. Обозначим для каждой пары $(a, \alpha) \in \mathscr{A}$ через $\mathscr{H}_{a, \alpha}$ подпространство в $A_{k}$, линейно порожденное операторами $\sigma(a, \alpha ; p, q),(p, q) \in$ $U(a, \alpha)$, т.е. подпространство операторов вида

$$
\kappa=\sum_{(p, q) \in U(a, \alpha)} c(p, q) \sigma(a, \alpha ; p, q)
$$

Назовем главной частью оператора (4.1) сумму членов с $p=0$, т.е. сумму

$$
\kappa^{0}=\sum_{(0, q) \in U(a, \alpha)} c(0, q) \sigma(a, \alpha ; p, q) \text {. }
$$

ТЕорема 1. Для любого оператора $\kappa^{0}$ вида (4.2) существует и притом единственный оператор $\kappa \in \mathscr{H}_{a, \alpha}$ с главной частью $\kappa^{0}$, принадлежсащий $A_{k}^{l}$.

Коэффициенты $c(p, q)$ в выражсении (4.1) для этого оператора имеют вид

$$
c(p, q)=\frac{c^{\prime}(p, q)}{p ! q !}
$$

где $p !=\prod_{i, j} p_{i j} !, q !=\prod_{i, j} q_{i j} !, a c^{\prime}(p, q)$ задаются следующими рекуррентными по $|p|=\sum_{i, j} p_{i j}$ соотношениями:

$$
c^{\prime}(p, q)=l c\left(p-e_{i j}, q+e_{i j}\right)+\sum_{i^{\prime}, j^{\prime}} q_{i^{\prime} j^{\prime}} c^{\prime}\left(p-e_{i j}, q+e_{i j^{\prime}}+e_{i^{\prime} j}-e_{i^{\prime} j^{\prime}}\right) \quad \text { npu } p_{i j}>0
$$

от выбора $(i, j)$ правая часть не зависит. 
ПРИмЕР. Оператор

$$
\kappa=\sum_{(p, q) \in U(a, \alpha)} \frac{1}{(|q|+l-1) ! p ! q !} \sigma(a, \alpha ; p, q),
$$

где $|q|=\sum_{i, j} q_{i j}$, принадлежит $A_{k}^{l}$.

В самом деле, для оператора $\kappa$ имеем

$$
c^{\prime}(p, q)=\frac{1}{(|q|+l-1) !}
$$

при любых $(p, q) \in U(a, \alpha)$ и легко убедиться, что эти коэффициенты удовлетворяют соотношениям (4.4).

ДОКАЗАТЕЛЬСТво ТЕОРЕМЫ 1. Пусть $\kappa$ - оператор вида (4.1). Очевидно, что при преобразованиях $x \mapsto g x, g \in G L(k)$, операторы (3.2) умножаются на $\operatorname{det} g$. Отсюда следует, что для любой функции $\varphi \in H_{k}^{l}$ дифференциальная форма $\kappa \varphi$ на $\mathscr{E}_{k, n}$ инвариантна относительно действия группы $G L_{+}(k)$. Поэтому условие, что $\kappa \in A_{k}^{l}$, эквивалентно условию, что эта форма горизонтальна на расслоении $\mathscr{E}_{k, n} \rightarrow G_{k, n}$. Так как каждьй вектор, касательньй к слою этого расслоения, есть $d g x, g \in G L(k)$, то это значит, что $\kappa \varphi$ сохраняется при замене $d x$ на $d x+d g x$.

Обозначим через $\widehat{\kappa}$ оператор, полученный из $\kappa$ заменой $d x$ на $d x+d g x$, и через $\widehat{\kappa}^{0}$ линейную относительно $d g$ часть этого оператора. Тогда условие $\kappa \in A_{k}^{l}$ эквивалентно условию $\widehat{\kappa}^{0} \varphi=0$ при $\varphi \in H_{k}^{l}$. Найдем коэффициенты $c(p, q)$ в выражении для $\kappa$, исходя из этого условия.

ЛЕмма. Если $\varphi \in H_{k}^{l}$, то для любих $j_{1}, \ldots, j_{N}, s_{2}, \ldots, s_{N}=1, \ldots, n u \nu=1, \ldots, k$ имеем

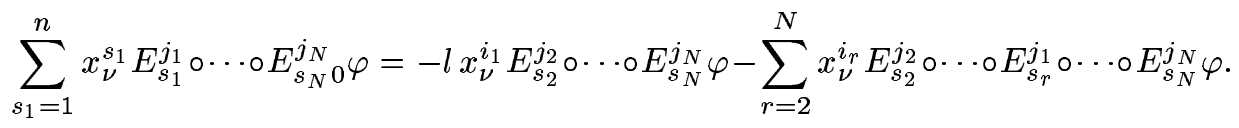

ДокАЗАТЕЛЬСтво. Так как $\varphi \in H_{k}^{l}$, то

$$
\sum_{s_{1}=1}^{n} x_{\nu}^{s_{1}} \partial_{s_{1}}^{\nu_{1}} \varphi=-l \delta_{\nu}^{\nu_{1}} \varphi
$$

Применив к этому равенству оператор $\partial_{s_{2}}^{\nu_{2}} \cdots \partial_{s_{N}}^{\nu_{N}}$, получим

$$
\sum_{s_{1}=1}^{n} x_{\nu}^{s_{1}} \partial_{s_{1}}^{\nu_{1}} \cdots \partial_{s_{N}}^{\nu_{N}} \varphi+\sum_{r=2}^{N} \delta_{\nu}^{\nu_{r}} \partial_{s_{2}}^{\nu_{2}} \cdots \partial_{s_{r}}^{\nu_{1}} \cdots \partial_{s_{N}}^{\nu_{N}} \varphi=-l \delta_{\nu}^{\nu_{1}} \partial_{s_{2}}^{\nu_{2}} \cdots \partial_{s_{N}}^{\nu_{N}} \varphi
$$

Умножив полученное равенство на $x_{\nu_{1}}^{j_{1}} \cdots x_{\nu_{N}}^{j_{N}}$ и просуммировав по $\nu_{1}, \ldots, \nu_{N}$, получаem (4.6).

В дальнейшем для краткости будем вместо $\sigma(a, \alpha ; p, q)$ писать $\sigma(p, q)$. Обозначим через $\widehat{\sigma}(p, q)$ оператор, полученньй из $\sigma(p, q)$ заменой $d x$ на $d x+d g x$, и через $\widehat{\sigma}^{0}(p, q)$ линейную относительно $d g$ часть оператора $\widehat{\sigma}(p, q)$. Из предложения 1 и леммы следует 
ПРЕДЛОЖЕНИЕ 2. Ограничение оператора $\widehat{\sigma}^{0}(p, q)$ на подпространство функиий $\varphi \in H_{k}^{l}$ имеет вид

$$
\begin{array}{r}
\widehat{\sigma}^{0}(p, q)=\sum_{i, j} p_{i j} \sigma^{i j}(p, q)-\sum_{i, j} q_{i j}\left(q_{i j}+l-1\right) \sigma^{i j}\left(p+e_{i j}, q-e_{i j}\right) \\
-\sum_{(i, j) \neq\left(i^{\prime}, j^{\prime}\right)} q_{i j^{\prime}} q_{i^{\prime} j} \sigma^{i j}\left(p+e_{i j}, q-e_{i j^{\prime}}-e_{i^{\prime} j}+e_{i^{\prime} j^{\prime}}\right),
\end{array}
$$

где $\sigma^{i j}(p, q)$ получается из $\sigma(p, q)=\bigwedge_{i=1}^{l} \sigma_{i}(p, q)$ заменой в $\sigma_{i}(p, q)$ одного столбиа $d x^{j}$ на столбеи, $d g x^{j}$.

СлЕДСТВИЕ. Озраничение оператора $\widehat{\kappa}^{0}$ на подпространство функиий $\varphi \in H_{k}^{l}$ имеет вид

$$
\begin{aligned}
\widehat{\kappa}^{0}= & \sum_{i, j} \\
& -\sum_{(p, q) \in U(a, \alpha)} p_{i j} c(p, q) \sigma^{i j}(p, q) \\
& -\sum_{(p, q) \in U(a, \alpha)} q_{i j}\left(q_{i j}+l-1\right) c(p, q) \sigma^{i j}\left(p+e_{i j}, q-e_{i j}\right) \\
& \left.\sum_{(p, q) \in U(a, \alpha)} q_{i j^{\prime}} q_{i^{\prime} j} c(p, q) \sigma^{\prime}\right) \neq(i, j)
\end{aligned}
$$

ЗАмечАниЕ. Операторы $\sigma^{i j}(p, q)$ не определены при $p_{i j}=0$. Однако выражения (4.7) и (4.8) корректны, поскольку $\sigma^{i j}(p, q)$ с $p_{i j}=0$ входят в эти выражения с коэффициентом 0. Аналогичное замечание относится и к последующим равенствам.

Положим

$$
c(p, q)=\frac{c^{\prime}(p, q)}{p ! q !} .
$$

ПРЕДЛОЖЕНИЕ 3. Условие, что форма кч, где $\varphi \in H_{k}^{l}$, опускается на грассманиан $G_{k, n}$, әквивалентно следуюшим соотношениям между коэффициентами onератора $\kappa$ :

$$
\begin{gathered}
c^{\prime}(p, q)=l c^{\prime}\left(p-e_{i j}, q+e_{i j}\right)+\sum_{i^{\prime}, j^{\prime}} q_{i^{\prime} j^{\prime}} c^{\prime}\left(p-e_{i j}, q+e_{i j^{\prime}}+e_{i^{\prime} j}-e_{i^{\prime} j^{\prime}}\right) \\
n p u p_{i j}>0, i=1, \ldots, l, j=1, \ldots, n .
\end{gathered}
$$

ДокАЗАТЕЛЬСтво. Условие, что $\kappa \varphi$ опускается на грассманиан $G_{k, n}$, эквивалентно соотношению $\widehat{\kappa}^{0}=0$. Значит, в силу (4.8)

$$
\begin{gathered}
\sum_{(p, q) \in U(a, \alpha)} p_{i j} c(p, q) \sigma^{i j}(p, q)=\sum_{(p, q) \in U(a, \alpha)} q_{i j}\left(q_{i j}+l-1\right) c(p, q) \sigma^{i j}\left(p+e_{i j}, q-e_{i j}\right) \\
+\sum_{(p, q) \in U(a, \alpha)} \sum_{\left(i^{\prime}, j^{\prime}\right) \neq(i, j)} q_{i j^{\prime}} q_{i^{\prime} j} c(p, q) \sigma^{i j}\left(p+e_{i j}, q-e_{i j^{\prime}}-e_{i^{\prime} j}+e_{i^{\prime} j^{\prime}}\right)
\end{gathered}
$$

при любых $i, j$, для которьх $p_{i j}>0$. 
Преобразуем (4.11) к виду

$$
\begin{aligned}
& \sum_{(p, q) \in U(a, \alpha)} p_{i j} c(p, q) \sigma^{i j}(p, q) \\
& =\sum_{(p, q) \in U(a, \alpha)} q_{i j}\left(q_{i j}+\sum_{s \neq i} q_{i s}+\sum_{s \neq j} q_{s j}+l-1\right) c(p, q) \sigma^{i j}\left(p+e_{i j}, q-e_{i j}\right) \\
& \quad+\sum_{(p, q) \in U(a, \alpha)} \sum_{i^{\prime} \neq i} \sum_{j^{\prime} \neq j} q_{i j^{\prime}} q_{i^{\prime} j} c(p, q) \sigma^{i j}\left(p+e_{i j}, q-e_{i j^{\prime}}-e_{i^{\prime} j}+e_{i^{\prime} j^{\prime}}\right) .
\end{aligned}
$$

Полученное равенство эквивалентно следуюшим соотношениям для коэффициентов $c(p, q)$ :

$$
\begin{aligned}
p_{i j} c(p, q)= & \left(q_{i j}+1\right)\left(q_{i j}+\sum_{s \neq i} q_{i s}+\sum_{s \neq j} q_{s j}+l\right) c\left(p-e_{i j}, q+e_{i j}\right) \\
& +\sum_{i^{\prime} \neq i} \sum_{j^{\prime} \neq j}\left(q_{i j^{\prime}}+1\right)\left(q_{i^{\prime} j}+1\right) c\left(p-e_{i j}, q+e_{i j^{\prime}}+e_{i^{\prime} j}-e_{i^{\prime} j^{\prime}}\right) \quad \text { при } p_{i j}>0 .
\end{aligned}
$$

Перейдя от $c(p, q)$ к коэффициентам $c^{\prime}(p, q)$, заданным равенством $(4.9)$, получаем

$$
\begin{aligned}
c^{\prime}(p, q)= & \left(q_{i j}+\sum_{s \neq i} q_{i s}+\sum_{s \neq j} q_{s j}+l\right) c^{\prime}\left(p-e_{i j}, q+e_{i j}\right) \\
& +\sum_{i^{\prime} \neq i} \sum_{j^{\prime} \neq j} q_{i^{\prime} j^{\prime}} c^{\prime}\left(p-e_{i j}, q+e_{i j^{\prime}}+e_{i^{\prime} j}-e_{i^{\prime} j^{\prime}}\right) .
\end{aligned}
$$

Полученные соотношения эквивалентны соотношениям (4.10).

Перейдем непосредственно к доказательству теоремы. Соотношения (4.10) выражают $c^{\prime}(p, q)$ при любом $|p|=s>0$ через $c^{\prime}(p, q)$ при $|p|=s-1$. Таким образом, при заданных $c^{\prime}(0, q),(0, q) \in U(a, \alpha)$, имеется не более одного решения системы (4.10). Чтобы доказать сушествование решения, достаточно убедиться, что равенства (4.10) корректно определяют $c^{\prime}(p, q)$, т.е. правые части этих равенств не зависят от выбора пары $(i, j)$, для которой $p_{i j}>0$.

Проведем доказательство индукцией по $s=|p|$. При $s=1$, т.е. когда $p=e_{k l}$ для некоторого $(k, l)$, утверждение очевидно. В самом деле, в этом случае имеется единственное соотношение вида (4.10), выражающее $c^{\prime}(p, q)$ через коэффициенты $c^{\prime}\left(0, q^{\prime}\right)$. Пусть теперь $|p|>1$, и пусть $p_{i j}>0$ и $p_{k l}>0,(i, j) \neq(k, l)$. Тогда в соответствии c (4.10) $c^{\prime}(p, q)$ можно определить двумя различными равенствами: равенством (4.10) и равенством

$$
c^{\prime \prime}(p, q)=l c^{\prime}\left(p-e_{k l}, q+e_{k l}\right)+\sum_{k^{\prime}, l^{\prime}} q_{k^{\prime} l^{\prime}} c^{\prime}\left(p-e_{k l}, q+e_{k l^{\prime}}+e_{k^{\prime} l}-e_{k^{\prime} l^{\prime}}\right)
$$

Нужно убедиться, что $c^{\prime \prime}(p, q)=c^{\prime}(p, q)$. 
В силу индуктивного предположения $c^{\prime}\left(p^{\prime}, q^{\prime}\right)$ при $\left|p^{\prime}\right|=|p|-1$ однозначно выражается по формулам (4.10) через $c^{\prime}\left(p^{\prime}, q^{\prime}\right)$ при $\left|p^{\prime}\right|=|p|-2$. Согласно этим формулам имеем

$$
\begin{aligned}
& c^{\prime}\left(p-e_{i j}, q+e_{i j}\right)=l c^{\prime}\left(p-e_{i j}-e_{k l}, q+e_{i j}+e_{k l}\right) \\
& \quad+\sum_{k^{\prime} l^{\prime}}\left(q_{k^{\prime} l^{\prime}}+\delta_{k^{\prime} l^{\prime}, i j}\right) c^{\prime}\left(p-e_{i j}-e_{k l}, q+e_{i j}+e_{k l^{\prime}}+e_{k^{\prime} l}-e_{k^{\prime} l^{\prime}}\right), \\
& c^{\prime}\left(p-e_{i j}, q+e_{i j^{\prime}}+e_{i^{\prime} j}-e_{i^{\prime} j^{\prime}}\right)=l c^{\prime}\left(p-e_{i j}-e_{k l}, q+e_{i j^{\prime}}+e_{i^{\prime} j}-e_{i^{\prime} j^{\prime}}+e_{k l}\right) \\
& +\sum_{k^{\prime} l^{\prime}}\left(q_{k^{\prime} l^{\prime}}+\delta_{k^{\prime} l^{\prime}, i j^{\prime}}+\delta_{k^{\prime} l^{\prime}, i^{\prime} j}-\delta_{k^{\prime} l^{\prime}, i^{\prime} j^{\prime}}\right) \\
& \quad \times c^{\prime}\left(p-e_{i j}-e_{k l}, q+e_{i j^{\prime}}+e_{i^{\prime} j}-e_{i^{\prime} j^{\prime}}+e_{k l^{\prime}}+e_{k^{\prime} l}-e_{k^{\prime} l^{\prime}}\right) .
\end{aligned}
$$

Подставив эти выражения в (4.10), получим после элементарных упрощений

$$
\begin{aligned}
c^{\prime}(p, q)= & l^{2} c^{\prime}\left(p-e_{i j}-e_{k l}, q+e_{i j}+e_{k l}\right)+l c^{\prime}\left(p-e_{i j}-e_{k l}, q+e_{i l}+e_{k j}\right) \\
& +\sum_{i^{\prime}, j^{\prime}} q_{i^{\prime} j^{\prime}}\left(l c^{\prime}\left(p-e_{i j}-e_{k l}, q+e_{i j^{\prime}}+e_{i^{\prime} j}-e_{i^{\prime} j^{\prime}}+e_{k l}\right)\right. \\
& +l c^{\prime}\left(p-e_{i j}-e_{k l}, q+e_{i j}+e_{i^{\prime} l}+e_{k j^{\prime}}-e_{i^{\prime} j^{\prime}}\right) \\
& +c^{\prime}\left(p-e_{i j}-e_{k l}, q+e_{i j^{\prime}}-e_{i^{\prime} j^{\prime}}+e_{i^{\prime} l}+e_{k j}\right) \\
& +c^{\prime}\left(p-e_{i j}-e_{k l}, q+e_{i^{\prime} j}-e_{i^{\prime} j^{\prime}}+e_{i l}+e_{k j^{\prime}}\right) \\
& \left.-c^{\prime}\left(p-e_{i j}-e_{k l}, q+e_{i j^{\prime}}+e_{i^{\prime} j}-2 e_{i^{\prime} j^{\prime}}+e_{i^{\prime} l}+e_{k j^{\prime}}\right)\right) \\
& +\sum_{i^{\prime}, j^{\prime}, k^{\prime}, l^{\prime}} q_{i^{\prime} j^{\prime}} q_{k^{\prime} l^{\prime}} c^{\prime}\left(p-e_{i j}-e_{k l}, q+e_{i j^{\prime}}+e_{i^{\prime} j}-e_{i^{\prime} j^{\prime}}+e_{k l^{\prime}}+e_{k^{\prime} l}-e_{k^{\prime} l^{\prime}}\right) .
\end{aligned}
$$

Правая часть полученного равенства симметрична относительно перестановки пар $(i j)$ и $(k l)$. Значит, то же выражение получается и для $c^{\prime \prime}(p, q)$, а потому $c^{\prime \prime}(p, q)=c^{\prime}(p, q)$.

5. Построение относительно замкнутых операторов. Пусть $\kappa^{0} \in A_{k}$-произвольный оператор вида

$$
\kappa^{0}=\bigwedge_{i=1}^{l}\left[x^{1}, \ldots, x^{r}, D^{\mu_{i, 1}}, \ldots, D^{\mu_{i, k-r}}\right], \quad \text { где } j_{i s} \leqslant r .
$$

В обозначениях п. $3 \kappa^{0}=\sigma\left(a, \alpha ; 0, q^{0}\right)$, где $a=\left\|a_{i s}\right\|$ и $q^{0}=\left\|q_{i s}^{0}\right\|-(l \times n)$-матрицы с элементами $a_{i s}=1$ при $s \leqslant r, a_{i s}=0$ при $s>r ; q_{i s}^{0}-$ число индексов $\mu_{i t}$, равных $s$; $\alpha=\left(\alpha_{1}, \ldots, \alpha_{r}, 0, \ldots, 0\right)$, где $\alpha_{s}=\sum_{i} q_{i s}^{0}$.

По теореме 1 в $\mathscr{H}_{a, \alpha}$ существует и притом единственный оператор $\kappa \in A_{k}^{l}$ с главной частью $\kappa^{0}$, а именно

$$
\kappa=\kappa^{0}+\sum_{\substack{(p, q) \in U(a, \alpha) \\ p \neq 0}} c(p, q) \sigma(a, \alpha ; p, q)
$$

где коэффищиенты $c(p, q)$ задаются формулами $(4.3),(4.4)$.

ТЕОРема 2. Oператор к является относительно замкнутым типа $r$. 
ДоКАЗАТЕЛьСТво. Для доказательства найдем ограничение дифференциальной формы $\kappa \varphi$, где $\varphi \in H_{k}^{l}$, на подмногообразие $G_{k, n}^{h} \subset G_{k, n} k$-подпространств, содержаших произвольное фиксированное $r$-мерное подпространство $h$.

Фиксируем в $h$ произвольньй базис $x_{i}=\left(x_{i}^{1}, \ldots, x_{i}^{n}\right), i=1, \ldots, r$. Не нарушая общности, можно предполагать, что $\Delta_{r}(x) \equiv \operatorname{det}\left\|x_{j}^{i}\right\|_{i, j=1, \ldots, r} \neq 0$.

Обозначим через $\widetilde{\mathscr{E}}_{k, n} \boldsymbol{x}$ подмногообразие матриц $x^{\prime} \in \mathscr{E}_{k, n}$, у которых первые $r$ строк совпадают с векторами $x_{i}$ фиксированного базиса в $h$ и, кроме того, $x_{j}^{i}=0$ при $i=1$, $\ldots, r$ и $j=r+1, \ldots, k$. Образ $\widetilde{\mathscr{E}}_{k, n}^{x}$ при отображении $\mathscr{E}_{k, n} \rightarrow G_{k, n}$ есть все подмногообразие $G_{k, n}^{h}$, а потому вместо формы $\kappa_{r} \varphi$ на $G_{k, n}^{h}$ можно рассматривать ее поднятие на многообразие $\widetilde{\mathscr{E}}_{k, n} \subset \mathscr{E}_{k, n}$.

ПРЕДЛОЖЕНИЕ 4. Для любой функиии $\varphi \in H_{k}^{l}$ имеем

$$
\kappa \varphi=\kappa^{0} \varphi \quad \text { нa } \tilde{\mathscr{E}}_{k, n}^{x}
$$

В самом деле, так как $d x^{i}=0$ на $\widetilde{\mathscr{E}}_{k, n}^{x}$ при $i \leqslant r$, то все слагаемые суммы $(5.2)$, кроме $\kappa^{0}$, равны нулю на $\widetilde{\mathscr{E}}_{k, n}$.

Заметим, что

$$
\left[x^{1}, \ldots, x^{r}, D^{\mu_{i, 1}}, \ldots, D^{\mu_{i, k-r}}\right]=\Delta_{r}(x) \sum\left(D_{r+1}^{\mu_{i, 1}^{\prime}} \wedge \cdots \wedge D_{k}^{\mu_{i, k-r}^{\prime}}\right)
$$

где суммирование ведется по всем перестановкам $\mu_{i, r+1}^{\prime}, \ldots, \mu_{i, k-r}^{\prime}$ индексов $\mu_{i, 1}, \ldots$, $\mu_{i, k-r}$. Отсюда следует

ПРЕДЛОЖЕНИЕ $5 . H a \widetilde{\mathscr{E}}_{k, n}^{x}$ u.меем

$$
\kappa^{0} \varphi= \pm\left(\Delta_{r}(x)\right)^{l} \sum_{\mu^{\prime}}\left(\bigwedge_{j=1}^{k-r} \bigwedge_{i=1}^{l} D_{r+j}^{\mu^{\prime} i j}\right) \varphi
$$

әде суммирование ведется по множеству $l \times(k-r)$-матрии, $\mu^{\prime}=\left\|\mu_{i j}^{\prime}\right\|$, получающихся из $\mu=\left\|\mu_{i j}\right\|$ всевозможными перестановками әлементов в каждой из строк.

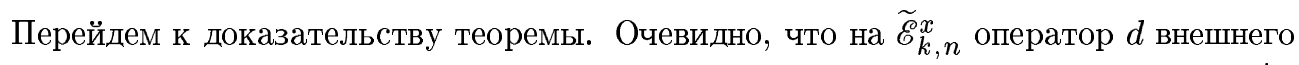
дифференцирования имеет вид $d=d_{r+1}^{r+1}+\cdots+d_{k}^{k}$. Так как каждый оператор $\bigwedge_{i=1}^{l} D_{r+j}^{\mu^{\prime} i j}$ представим на $\widetilde{\mathscr{E}}_{k, n}^{x}$ как линейная комбинация операторов вида $\bigwedge_{i=1}^{l} d_{r+j}^{s_{i}}$ с коэффициентами, зависящими только от $x$, то в силу (1.6)

$$
d_{r+j}^{r+j}\left(\bigwedge_{i=1}^{l} D_{r+j}^{\mu^{\prime} i j}\right) \varphi=0
$$

для любой функции $\varphi$ из образа $\mathscr{J}_{k l}$. Значит, в силу предложений 4 и $5 d(\kappa \varphi)=$ $d\left(\kappa^{0} \varphi\right)=0$ на $\widetilde{\mathscr{E}}_{k, n}^{x}$. 
6. Формулы обращения. Обозначим через $C_{q}$ пространство относительно замкнутых операторов типа $l$ и ранга $q<(k-l)(n-k)$. Если $\kappa \in C_{q}$, то для любого $h \in G_{l, n}$ и любого $q$-мерного цикла $\gamma^{h} \subset G_{l, n}^{h}$ имеем

$$
\int_{\gamma^{h}} \kappa\left(\mathscr{J}_{k l} f\right)=c\left(\gamma^{h}\right)(L f)(x)
$$

где $c\left(\gamma^{h}\right)$ зависит только от класса гомологий $\gamma^{h}, L \in \mathscr{D}$, a $x \in \mathscr{E}_{l, n}$ - любой элемент из прообраза $h$. В нетривиальном случае, т.е. когда $c\left(\gamma^{h}\right) \not \equiv 0$, равенство (6.1) задает гомоморфизм

$$
\pi: C_{q} \rightarrow \mathscr{D}
$$

перестановочньй с представлениями группы $G L(n)$, действующими в пространствах $C_{q}$ и $\mathscr{D}$. Его ядром является по определению подпространство $Z_{q} \subset C_{q}$ тривиальных операторов.

Так как правая часть в (6.1) зависит только от образа $h \in G_{l, n}$ точки $x$, то при преобразованиях $x \mapsto g x, g \in G L(l)$, операторы $L$ из образа $C_{q}$ умножаются на $(\operatorname{det} g)^{k}$.

Опишем подпространство $C_{q}^{0}$ операторов $\kappa \in C_{q}$, для которых $L$ - оператор нулевой степени, т.е. равенство(6.1) имеет вид

$$
\int_{\gamma^{h}} \kappa\left(\mathscr{J}_{k l} f\right)=c\left(\gamma^{h}\right) P(x) f(x),
$$

где $P(x)$ - полином от элементов матрицы $x \in \mathscr{E}_{l, n}$, удовлетворяющий условию

$$
P(g x)=(\operatorname{det} g)^{k} P(x) \quad \text { для любого } g \in G L(k) .
$$

Обозначим через $\mathscr{H}_{l}^{k}$ пространство всех полиномов $P(x)$, удовлетворяющих условию (6.3), т.е. пространство однородных полиномов степени $k$ от миноров порядка $l$ матриц $x \in \mathscr{E}_{l, n}$.

ТЕОрема 3. Если $k+l<n u k-l$ четно, то при $q=l(k-l)$ отображсение $\kappa \mapsto P(x)$, определенное равенством (6.2), является изоморфизмом пространств:

$$
C_{q}^{0} / Z_{q}^{0} \simeq \mathscr{H}_{l}^{k}
$$

где $Z_{q}^{0}$ - подпространство тривиальных операторов. При этом отображснии прообразом полинома $\left(\Delta_{l}(x)\right)^{k}$, где $\Delta_{l}(x)=\operatorname{det}\left\|x_{j}^{i}\right\|_{i, j=1, \ldots, l}$, является с точностью до мнохителя оператор $\kappa \in A_{k}^{l}$ с главной частью

$$
\kappa^{0}=\bigwedge_{i=1}^{l}[x^{1}, \ldots, x^{l}, \underbrace{D^{i}, \ldots, D^{i}}_{k-l}]
$$


ДоКАЗАТЕЛЬСтво. Известно, что пространство $\mathscr{H}_{l}^{k}$ неприводимо относительно действия групшы $G L(n)$. Поэтому так как отображение $C_{q}^{0} \rightarrow \mathscr{H}_{l}^{k}$ перестановочно с представлениями грушы $G L(n)$ в пространствах $C_{q}^{0}$ и $\mathscr{H}_{l}^{k}$, то образ $C_{q}^{0}$ есть либо 0 и тогда $C_{q}^{0}=Z_{q}^{0}$, либо все пространство $\mathscr{H}_{l}^{k}$ и тогда $C_{q}^{0} / Z_{q}^{0} \simeq \mathscr{H}_{l}^{k}$.

Итак, для доказательства теоремы достаточно убедиться, что если $\kappa$-оператор с главной частью $(6.4)$ и $\varphi=\mathscr{J}_{k l} f$, то

$$
\int_{\gamma^{h}} \kappa \varphi=c\left(\gamma^{h}\right)\left(\Delta_{l}(x)\right)^{k} f(x)
$$

где $c\left(\gamma^{h}\right) \not \equiv 0$.

Из (6.4) и (5.2) следует, что на $\widetilde{\mathscr{E}}_{k, n}^{x}$ имеем

$$
\kappa \varphi=\kappa^{0} \varphi=[(k-l) !]^{l}\left(\Delta_{l}(x)\right)^{l} \bigwedge_{j=1}^{k-l} \bigwedge_{i=1}^{l} D_{l+j}^{i}=[(k-l) !]^{l} \bigwedge_{j=1}^{k-l}\left(\Delta_{l}(x)\right)^{k} \bigwedge_{i=1}^{l} d_{l+j}^{i} .
$$

Значит,

$$
\kappa^{0} \varphi=c\left(\Delta_{l}(x)\right)^{k} \kappa_{l} \quad \text { на } \widetilde{\mathscr{E}}_{k, n}^{x},
$$

где $\kappa_{l}$ - оператор, заданный равенством (1.7). Соотношение (6.5) следует непосредственно из формулы обращения (1.8), связанной с оператором $\kappa_{l}$.

\section{СПИСОК ЦИТИРОВАННОЙ ЛИТЕРАТУРЫ}

[1] Гельфанд И. М., Гиндикин С. Г., Граев М. И. Интегральная геометрия в афффинном и проективном пространствах // Итоги науки и техники. Соврем. пробл. матем. Т. 16. М.: ВИНИТИ, 1980. С. 53-226.

[2] Гельфанд И.М., Гиндикин С. Г., Граев М. И. Избранные задачи интегральной геометрии. М.: Добросвет, 2000.

[3] Гельфанд И. М., Граев М. И. Описание всех формул обращения в задаче интегральной геометрии, связанной с грассманианом $G_{k, n} / /$ Препринт ин-та прикл. математики АН СССР. № 102, 1986. C. 1-27.

[4] Graev M. Intermediate inversion formulas in integral geometry // Preprint ESI. № 630, 1998. P. 1-14.

[5] Граев М. И. Задача интеграљной геометрии, связанная с тройкой грассмановых многообразий // Функцион. анализ и его прилож. 2000. Т. 34. № 4.

[6] Гельфанд И.М., Граев М.И., Шапиро З.Я. Задача интегральной геометрии, связанная с парой грассмановых многообразий // Докл. АН СССР. 1970. Т. 193. № 2. С. 259-262.

[7] Гельфанд И. М., Граев М. И., Шапиро З. Я. Интегральная геометрия в проективном пространстве // Функцион. анализ и его прилож. 1970. Т. 4. №1. С. 14-32.

Научно-исследовательский институт системных исследований РАН, г. Москва

E-mail: mgraev@niisi.msk.ru

Поступило 31.08 .2000 